QK321.4

. $\mathrm{P} 7$

Procopianu-Procopovici, A

Floristisches aus den Gebirgen

der Bukowina 


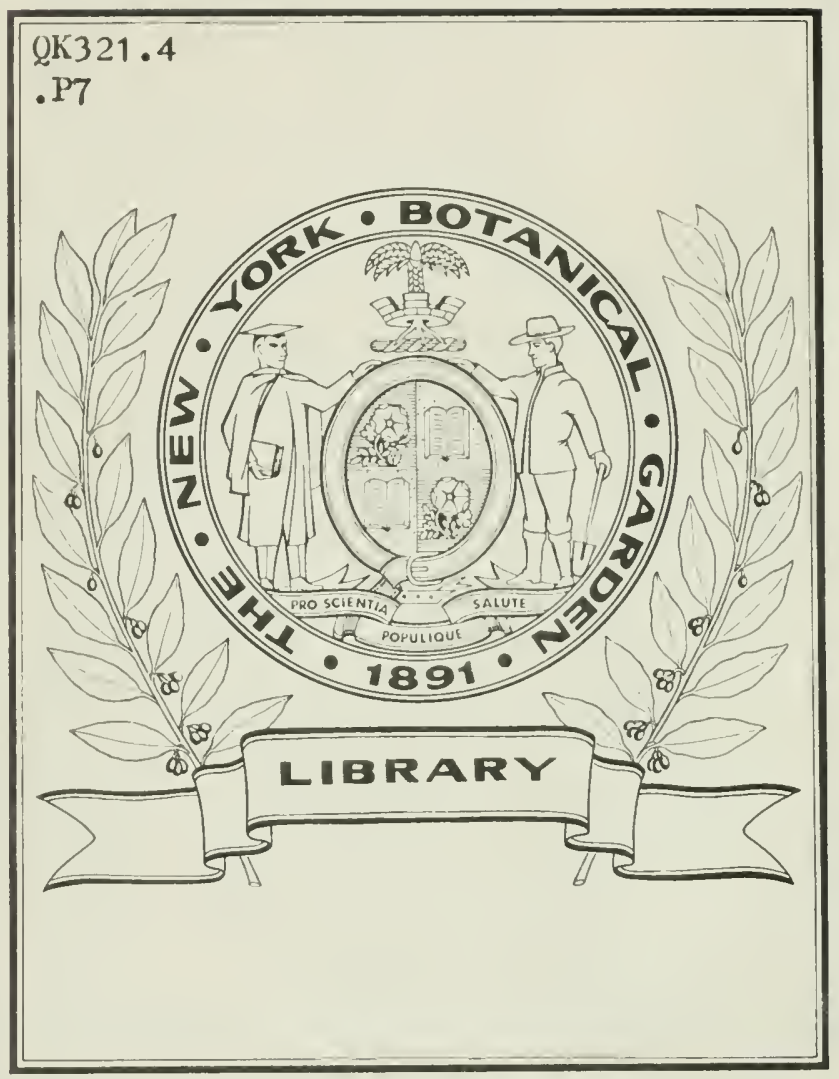






\title{
Floristisches aus den Gebirgen der Bukowina.
}

\author{
Von \\ A. Procopiamu-Procopovici.
}

(Vorgelegt in der Versammlung am 8. Jinner 1890.)

Weil die rollständige Bearbeitung des hener anf meinen botanischen kxcursionen gesammelten Materials und eine Veröftentlichung aller dabei gewonnenen Beobachtungen nicht so bald statt haben kann, so erlatube ich mir im Folgenden Bemerkungen nur an etliche anserwählte Pfanzen ans dem höheren Vorgebirge und aus der subalpinen Region der Bukowina anzukü̈pfen, welche insofern ein besonderes Interesse darbieten, als darin für das Gebiet neue, beziehungsweise zweifelhafte Fundorte oder Pflanzen allein Berïcksichtigung fanden

Für das Gebiet nene Pflanzen sind mit einem* bezeichnet.

Mit ! werden Fundortsangaben bestätigt.

* Phleum IIichclii All. Kommt bloss anf einer subalpinen Wiese am Riueu: Curmătura, ca. $1550 \mathrm{~m}$ hoch, vor.

Carex atrata L. Auf subalpinen Wiesen am Rareu und Todiresen, fermer am Zajul.

* Carex tristis M. B. Mit der vorigen vergesellschaftet am Rareu und Todiresen beobachtet.

Allium fallax Don. Hier und da anf Kalk; am Raren und Suhard, sowie auf Piatra-Cibon.

Allium Victorialis L. Kommt am Rareu! (Herbich, Flora der Bukowina, S. 73), aber auch am Sulard vol.

* Muscari transsilvanicum Schur. Nur auf den subalpinen Wiesen des Rareu und Todireseu.

Salix pentandra L. (ron Herbich in der Einleitung zur Flora der Bukowina, S. 4 olne Fundortangabe verzeichnet) und Pinus uliginosa Neum. kommen anf den Torfmooren bei Dorna-Vatra! und Dorna-Candreni! (nach Porcius, Flora distr. Năsěud. in Zeitschr. Transilvania, 1881, S. 116, resp. S. 117), ferner anch bei Poiana-Stampei vor.

Betula pubescens Ehrh. Ebenfalls auf den Torfmooren bei Dorna-Vatra und Poiana-Stampei.

Thesium alpinum L. Anf Wiesen in Gesellschaft von Arnica montana und Vaccineen stellenweise nicht selten; am Raren! (Herbich, l. c., S. 131), Todiresen, anf der Fluturica, am Suhard und Rume bei Vatra-Dornei.

* Orobanche transsileanica Poreins (1. c., 1881, S. 121). Zumeist auf Thymus in der subalpinen und oberen Bergregion zerstreut; am Zapul, Jednl, Lucaci, Dorna: Runc.

* Rhinanthus alpinus Baumg. In der subalpinen Region am Zapul und Suhard. 
I'cdicularis exaltata Bess, * var. carpatica Porcius. Auf subalpinen Wiesen an Rareu! (Herbich, l. c., S. 276 ; vgl. Kualup, Ptl. Gal. u. d. Bukow., s. 283 und P'orcius, l. c., 1881, S. 121) und l'odirescu, sowie am Zalpul und Jedul.

Eritrichum villosum Bunge kommt anssel auf der Kalkilpe Pietrile-Doamuei! (Herbieh, l. e., S. 252) anch am Rar'u und 'T'odiresen vor, aber überall nur anf Felsen.

* Myosotis alpestris Schmilt. In der subalpinen Region auf Wiesen; am T'odirescu, liaren und Pietrile-Doammei.

Pulmonaria rubra Sehott. In Krummholze am Rauen und Todireseu.

* Gentiana caucasica MI. B. Ist auf somigen WViesen im höheren Vorgebirge und in der subalpinen Region allgemein verbreitet.

Gentiana excisa Prex. und nieht Gentiana acantis L. (rgl. Knap ), 1. c., S. 187, 188) kommt auf der Fluturiea (Bauer exs.), am Raren und Todiresen vor.

Camponula rbictina Gr. et Sch. Im Krummholze nicht selten; im Giumaleu!

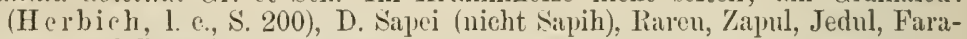
oane und Lucaci: Battea-mare.

Aster culpinus I. Auf subalpinen Kalkfelsen, nur an 'Todirescu, Rareu und Pictrile-Dommei (Herbich, 1. c., s. 143).

Anthemis temifolice Schm. Kommt auf Pietrile-Doamnei! (Herbich, 1. e., S. 157), aber anch an liareu: Parrete, in nörllicher Exposition vor.

* Tephroseris micromhiza Schur = Cineraria longifolia Jaeq. $\beta$. sulphurea Baumgt. Im Krummbolze am Rareu und Todirescu selten.

* Cirsium decussctum Janka. Rnderal um Semmhïtten und am Wege; am Oușor, Todirescu und Rareu.

Centaurea montana L. Auf somigen Wiesen, mit Vorliebe auf Kalk; am Rareu und Torlirescn, bei Ciocănesci und Cărlibaba, auf der Flıturica, an Jedul mud Lapul.

Crepis Jacquinii Tausch, Auf Kalkfelsen der subalpinen Region; nicht nur anf Pietrile-Doamnei! (Herbich, 1. c., S. 191), sondern auch am Rareu, Todiresen und hapul.

* Galium aristatum L. In trockenercn Wäldern der Vorgebirgsregion überall. (Steigt auf der Nordseite ron Pietrile-Doammei bis ca. $1575 \mathrm{~m}$ hinauf.) Galium silvaticum L. (Kü] l, 1. e., S. 179 und Herbich, 1. c., S. 209) kommt bei uns - wenigstens im Vorgebirge - nirgends vor.

Selum glaucum W. Kr. Auf Kalkfelsen mul im Kalkschutt im oberen Vorgebirge und in der subalpinen Region; am Zapul! bei Carlibaba! (Herbich, 1. c., S. 312), auf der Fluturica! (Baner exs.), Ciocănesci und Pojorita.

Rubus saxatitis L. In Krummholze am Rareu, selten, $1600 \mathrm{~m}$.

* Gercuium alpestre Schur. Auf subalpinen Wiesen auf Pietrile-Doammei!, am Rareu! (Herbich, 1. e., S. 411, unter Geranium sileaticum L.; vgl. Poreins, 1. c., 1882, S. 17) und 'Todireseu, ferner an Jedul.

Hypericum alpinum W. K. Am Raren im Krummholze, selten, $1600 \mathrm{~m}$.

Erysimum Witmanii Kaw. Auf Kalkfelsen und in Kalkschutt am Piatra-Cibou!, Jerlul! (Herbieh exs., Kna l' J, 1. e., S. 307) und Katjul, bei Cărlibaba und Ciocănesei, anf' Jietrile-Doamuei! (Herbieh exs., s. U.), am Rareu! (Kawalzki, Enmmeratio, 1. 30, nuch Knapp, l. c.) und Todirescu.

* Aquilegia glandulosa Fisch. Auf sonnigen Wiesen der subalpinen Tiegion; am Ritren und 'Todireseu, aut' der Fluturica. (Vgl. Herbich, l. e., S. 332 und Ku a p]', l. c., S. 293.) 


OK 321.4.P7 $7^{\text {Nork Botanicel Garden Library }}$

Procoplanu-Procopov/Floristisches aus de

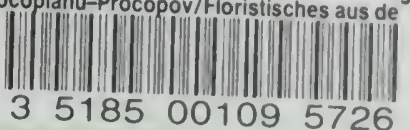


$-$ 